\title{
Numerical Methods for Quasicrystals
}

\author{
Kai Jiang, 2,a) and Pingwen Zhang ${ }^{1, b)}$ \\ 1) LMAM, CAPT and School of Mathematical Sciences, Peking University, \\ Beijing 100871, P.R. China \\ ${ }^{2)}$ Hunan Key Laboratory for Computation and Simulation in \\ Science and Engineering, Xiangtan University, Xiangtan 411105, \\ P.R. China
}

(Dated: 28 August 2018)

Quasicrystals are one kind of space-filling structures. The traditional crystalline approximant method utilizes periodic structures to approximate quasicrystals. The errors of this approach come from two parts: the numerical discretization, and the approximate error of Simultaneous Diophantine Approximation which also determines the size of the domain necessary for accurate solution. As the approximate error decreases, the computational complexity grows rapidly, and moreover, the approximate error always exits unless the computational region is the full space. In this work we focus on the development of numerical method to compute quasicrystals with high accuracy. With the help of higher-dimensional reciprocal space, a new projection method is developed to compute quasicrystals. The approach enables us to calculate quasicrystals rather than crystalline approximants. Compared with the crystalline approximant method, the projection method overcomes the restrictions of the Simultaneous Diophantine Approximation, and can also use periodic boundary conditions conveniently. Meanwhile, the proposed method efficiently reduces the computational complexity through implementing in a unit cell and using pseudospectral method. For illustrative purpose we work with the Lifshitz-Petrich model, though our present algorithm will apply to more general systems including quasicrystals. We find that the projection method can maintain the rotational symmetry accurately. More significantly, the algorithm can calculate the free energy density to high precision.

\footnotetext{
a) Electronic mail: kaijiang@xtu.edu.cn

b) Electronic mail: pzhang@pku.edu.cn
} 


\section{INTRODUCTION}

As early as the 1890s, the periodic structures (crystals) in three dimensions were determined by 230 space groups based on periodicity, and then the classical crystallography was completed, in which the allowed rotational symmetry is only 1-, 2-, 3-, 4-, 6-fold symmetry. Both the structure determination and the study of physical properties are based on the periodicity which allows the study to be simplified to a unit cell. However, in the 1980s, a forbidden 5 -fold symmetry electron diffraction pattern was discovered by Shechtman et al. 1 in a rapid cooled Al-Mn alloy. Later, the term "quasicrystals" appeared for the first time to describe the non-conventional ordered structures ${ }^{2}$. In an idealized description, quasicrystals have quasiperiodic, rather than periodic, translational order with non-crystallographic symmetry. In the early theory of tilings, the discovery of aperiodic tiling with 5-fold symmetry of the plane by Penrose ${ }^{\frac{\sqrt[314]{4}}{3}}$ showed that such well-ordered systems were mathematically possible. Since the original discovery, hundreds of quasicrystals have been reported and confirmed in metallic alloys with 5-, 8-, 10-, 12- fold orientational symmetry $\underline{[5]}$. Two decades after the first discovery of quasicrystals in metallic alloys, several soft quasicrystals have been found in soft matter systems ${ }^{7-10}$. The building blocks of the solid-state quasicrystals are the atoms or small molecular on the atomic scale, whilst the building blocks of soft quasicrystals are on a much larger scale of ten to hundreds of nanometers. Therefore the continuous density distribution are more appropriate for studying soft quasicrystals. Accordingly the coarsegrained free energy of density functions have been widely applied to treat phases and phase transitions, especially for soft matter systems 11,13 .

Quasicrystals are one kind of space-filling structures. Two kinds of theoretical approaches have been developed to study quasicrystals, or more generally, aperiodic crystals. The first method is an approximate approach which studies crystalline approximants rather than quasicrystals. The crystalline approximants are periodic structures in which the arrangements of lattices closely approximate the local structures in quasicrystals. Many crystalline approximants related to quasicrystals have also been discovered 14 . These approximants may play an important role in describing the local structures of quasicrystals, their formation, stability, and physical properties. The second approach is a direct method to study the quasicrystals or aperiodic crystals in the hyperspace, called the higher-dimensional approach. From this approach, a quasiperiodic structure may be viewed as a periodic structure by ex- 
tending it into a higher-dimensional space. Its symmetries can be expressed in terms of the conventional point groups and space groups of higher-dimensional periodic crystals $15[16$. In the higher-dimensional description, quasiperiodic structures result from irrational physicalspace cuts of appropriate periodic hypercrystal structures. It is so-called cut-and-project method. The higher-dimensional approach reveals the hidden structural correlations. To implement the higher-dimensional approach in the direct space one must know the discrete lattice arrangements of higher-dimensional periodic structure. The embedded spaces of $d$ dimensional quasiperiodic structures are abstract spaces whose dimensions are more than three. The dimensions of the embedded space are dependent on the symmetry of the quasicrystal $(d>1)$ 16117. For example, the quasicrystals with 5-, 8-, 10-, and 12-fold symmetry need to be embedded into four-dimensional space. While for the quasiperiodic structures with 7-, 9-, 18-fold symmetry, the dimension of the embedding spaces increases to six. For other symmetries, embedding spaces with even higher dimension will be needed. Therefore it makes the method difficult for implementing. Another disadvantage of applying the higherdimensional approach in direct space is that in practical problems one is required to compute the continuous quasiperiodic distribution rather than the discrete quasiperiodic lattice. A convenient way to describe the quasiperiodic structures is in the higher-dimensional reciprocal space $\frac{16}{16}$. By redefining point-group symmetry and space-group symmetry in terms of gauge functions, a broader Fourier-space crystallography $[1820$ has also been developed to describe quasiperiodic structures.

The traditional idea for treating quasicrystals is using a periodic structure to approximate the quasiperiodic structure. The method has been applied to molecular dynamics simulations $^{21} 24$, Monte Carlo simulations ${ }^{25}$, numerical discretization methods 26 . In other words, those methods compute crystalline approximants rather than quasicrystals. A natural expectation is that the obtained approximants should approximate quasicrystals as the computational box goes to infinity. The advantage of the approximate method is that using the periodic boundary conditions is convenient. However, the approach can not obtain quasicrystals exactly unless the computational box is the full space because of the restriction of the Simultaneous Diophantine Approximation (SDA) 27. We will further explain this point in Sec.III. In this paper, we focus on the development of numerical methods for generating quasicrystals to high precision, rather than crystalline approximants. The proposed approach is based on the observation that the Fourier spectrum of a $d$-dimensional quasicrys- 
tal, consists of $\delta$ peaks on a $\mathbb{Z}$-module, $\mathbf{k}=\sum_{i=1}^{n} h_{i} \mathbf{p}_{i} \in \mathbb{R}^{d}, h_{i} \in \mathbb{Z}$, of rank $n(n>d)$ with basis vectors $\mathbf{p}_{i}{ }^{15 / 16}$. Therefore, an natural idea is directly computing the Fourier spectrum of quasicrystals instead of using periodic cells to compute crystalline approximants in real space.

\section{LIFSHITZ-PETRICH MODEL}

Although our proposed method is applicable to any model including quasicrystals. For illustrative purpose we utilize the Lifshitz-Petrich model ${ }^{26}$ to demonstrate our algorithm. Before we go further, a short introduction to the Lifshitz-Petrich model is necessary. LifshitzPetrich model is a coarse-grained mean-field theory. It is specially appropriate for studying the phase behaviour of soft matters involving quasicrystals 1211328 . In particular, the LifshitzPetrich free free energy density functional is

$$
F[\phi(\mathbf{r})]=\frac{1}{V} \int d \mathbf{r}\left\{\frac{c}{2}\left[\left(\nabla^{2}+1\right)\left(\nabla^{2}+q^{2}\right) \phi\right]^{2}-\frac{\varepsilon}{2} \phi^{2}-\frac{\alpha}{3} \phi^{3}+\frac{1}{4} \phi^{4}\right\} .
$$

In Eqn. (1), $\phi(\mathbf{r})$ is the order parameter. $V$ is the system volume. $q$ is an irrational number depending on the symmetry. $\varepsilon$ is the reduced temperature. $c>0$ is an energy penalty to ensure that the principle reciprocal vectors of structures is located on $|\mathbf{k}|=1$ and $|\mathbf{k}|=q$. $\alpha>0$ is a phenomenological parameter. For quasicrystals the system volume $V$ should go to infinity since quasicrystals are the space-filling structures without periodicity. The most significant feature of the Lifshitz-Petrich model is the existence of two characteristic length scales, 1 and $q$, which is a necessary condition to stabilize the quasicrystals $26 \mid 29$. Therefore it is a suitable model to demonstrate our proposed approach.

Theoretically, the ordered patterns, including periodic and quasiperiodic, are corresponding to local minima of the free energy functional of the system with respect to order parameter $\phi$. Accordingly the order parameter $\phi^{*}$ is the minimum of the free energy density functional, which means

$$
\left.\frac{\delta F}{\delta \phi(\mathbf{r})}\right|_{\phi^{*}}=0 .
$$

In order to find the equilibrium state, we introduce a relaxational dynamical to minimize the Lifshitz-Petrich energy functional which yields

$$
\frac{\partial \phi}{\partial t}=-\frac{\delta F}{\delta \phi}=-c\left(\nabla^{2}+1\right)^{2}\left(\nabla^{2}+q^{2}\right)^{2} \phi+\varepsilon \phi+\alpha \phi^{2}-\phi^{3} .
$$


We choose a semi-implicit scheme to solve the dynamical equation (3):

$$
\frac{1}{\Delta t}\left(\phi_{t+\Delta t}-\phi_{t}\right)=\varepsilon \phi_{t}-c\left(\nabla^{2}+1\right)^{2}\left(\nabla^{2}+q^{2}\right)^{2} \phi_{t+\Delta t}+\alpha\left(\phi^{2}\right)_{t}-\left(\phi^{3}\right)_{t}
$$

$\Delta t$ is the time step size. The specific implementation of the semi-implicit method for different numerical methods will be discussed in Sec.III. The current paper is devoted to develop a numerical method to calculate quasicrystals rather than crystalline approximants.

\section{NUMERICAL METHODS}

\section{A. Crystalline Approximant Method (CAM)}

\section{Method Description of $C A M$}

Numerical methods designed for periodic structures have been used to study quasicrystals approximately 26 . Here we call this method the "crystalline approximant method (CAM)". In order to describe this method, a brief introduction of numerical methods for treating periodic structures is necessary, more details can be found in 30 . For any $d$-dimensional periodic function $f(\mathbf{r}), \mathbf{r} \in \mathbb{R}^{d}$, the repeated structural unit is called a unit cell. A primitive unit cell, described by $d$ vectors $\mathbf{e}_{1}, \mathbf{e}_{2}, \ldots, \mathbf{e}_{d}$, has the smallest possible volume. The Bravais lattice vector is then defined by

$$
\mathbf{R}=l_{1} \mathbf{e}_{1}+l_{2} \mathbf{e}_{2}+\cdots+l_{d} \mathbf{e}_{d}
$$

where $\mathbf{l}=\left(l_{1}, l_{2}, \ldots, l_{d}\right)$ is a $d$-dimensional vector with components $l_{i} \in \mathbb{Z}$. For any $\mathbf{R}$ in the Bravais lattice, the structure is invariant under a lattice translation, i.e., $f(\mathbf{r}+\mathbf{R})=f(\mathbf{r})$. Given the primitive vectors $\left(\mathbf{e}_{1}, \mathbf{e}_{2}, \ldots, \mathbf{e}_{d}\right)$, the primitive reciprocal vectors $\left(\mathbf{e}_{1}^{*}, \mathbf{e}_{2}^{*}, \ldots, \mathbf{e}_{d}^{*}\right)$ satisfy the equation

$$
\mathbf{e}_{i} \cdot \mathbf{e}_{j}^{*}=2 \pi \delta_{i j}
$$

The reciprocal lattice vector is then specified by

$$
\mathbf{k}=k_{1} \mathbf{e}_{1}^{*}+k_{2} \mathbf{e}_{2}^{*}+\cdots+k_{d} \mathbf{e}_{d}^{*}
$$

where $k_{i} \in \mathbb{Z}$. One of the most important properties of the reciprocal lattices is that plane

waves $\left\{e^{i \mathbf{k} \cdot \mathbf{r}}\right\}$ form a set of basis functions for any function with periodicity of the lattice. 
The periodic function $f(\mathbf{r})$ on the Bravais lattice can be expanded as

$$
f(\mathbf{r})=\sum_{\mathbf{k}} \hat{f}(\mathbf{k}) e^{i \mathbf{k} \cdot \mathbf{r}} .
$$

For periodic structures, the reciprocal lattice vectors have two important features: $\mathbf{e}_{1}^{*}, \mathbf{e}_{2}^{*}$, $\ldots, \mathbf{e}_{d}^{*}$ are linearly independent; and $k_{i} \in \mathbb{Z}, i=1,2, \ldots, d$. In the direct space, the lattice vectors have the same properties.

The main idea of CAM is to use periodic structures to approximate quasicrystals. For a $d$-dimensional quasicrystals, its reciprocal lattice vectors $\mathbf{k}$ can be expressed by $d$ linearly independent reciprocal vectors, $\mathbf{e}_{1}^{*}, \mathbf{e}_{2}^{*}, \ldots, \mathbf{e}_{d}^{*}$,

$$
\mathbf{k}=p_{1} \mathbf{e}_{1}^{*}+p_{2} \mathbf{e}_{2}^{*}+\cdots+p_{d} \mathbf{e}_{d}^{*}
$$

It is important to note that the $p_{i} \in \mathbb{R}$, usually including irrational numbers, that is, $\mathbf{k}$ can not be represented by linear combinations of $\mathbf{e}_{i}^{*}$ with integer-valued coefficients. However, the quasiperiodic function $\phi(\mathbf{r})$ can be expanded as in the following form

$$
\phi(\mathbf{r})=\sum_{\mathbf{k}} \hat{\phi}(\mathbf{k}) e^{i \cdot(L \mathbf{k}) \cdot \mathbf{r} / L}, \quad \mathbf{r} \in[0,2 \pi L)^{d}
$$

If there exists a rational number $L$ such that $L p_{i} \in \mathbb{Z}$ or $L p_{i}$ can be made arbitrarily close to a series of integers, for all $i=1,2, \ldots, d$ and $\mathbf{k}$, which means

$$
\left|L \cdot\left(p_{1}, p_{2}, \ldots, p_{d}\right)-\left(\left[L p_{1}\right],\left[L p_{2}\right], \ldots,\left[L p_{d}\right]\right)\right|_{l^{\infty}} \rightarrow 0, \text { for all } \mathbf{k}
$$

where [.] rounds the number · to the nearest integer. Then numerical methods designed for periodic structures can be used to treat quasicrystals. Without loss of generality, we can always choose $(1,0, \ldots, 0)$ as one of the primitive reciprocal vectors. Therefore, the rational number $L$ becomes an integer. The problem of determining $L$ is a well-known problem, which deals with the approximation of real numbers by rational numbers or integers, called the Simultaneous Diophantine Approximation (SDA) in number theory 27 . In view of numerical computability, the integer $L$ should be as small as possible. For simplicity, we denote the approximate error of SDA as $E_{S D A}$.

From the above description, the condition of SDA must be satisfied for the implementation of CAM. Therefore, there are two sources of errors of the CAM, from the discretization, and from the Diophantine approximation. The integer $L$ is dependent on these irrational 
numbers $p_{i}$ due to rotational symmetry and the desired precision of the approximation. In the subsequent numerical examples (in Sec.III C), we will find that $L$ increases very quickly as the desired precision becomes higher. This greatly increases the computational cost. For CAM, the computational box should satisfy the condition (11). Therefore, the edge length $D_{\mathbf{k}}$ of computational box is close to $D_{\mathbf{k}}=L \times 2 \pi$, or $D=n \cdot L \times 2 \pi, n \in \mathbb{N}^{+}$.

\section{An Example of 12-fold Rotational Symmetry}

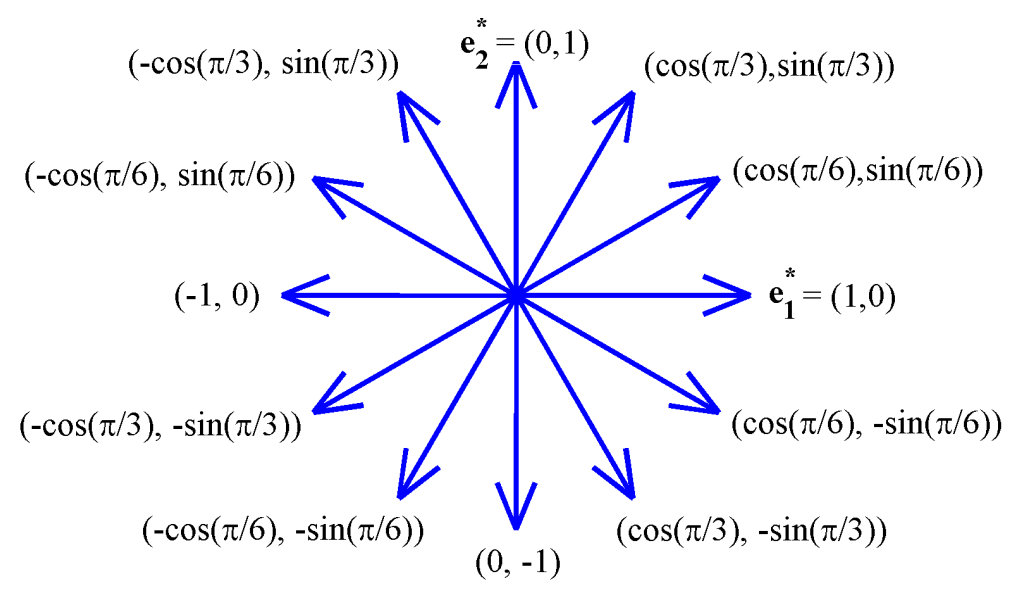

FIG. 1: Sets of reciprocal lattice vectors $\{\mathbf{k}\}$ of 12 -fold rotational symmetry in 2-dimensional space.

In order to make the above method clearer, we take an example of 12-fold rotational symmetry in two-dimensions to demonstrate this point. A schematic plot in the reciprocal space is shown in Fig. 1 . Let two noncollinear vectors $\mathbf{e}_{1}^{*}=(1,0)$ and $\mathbf{e}_{2}^{*}=(0,1)$ comprise the primitive reciprocal vectors, other reciprocal vectors of the 12-fold case are represented as linear combinations of $\mathbf{e}_{1}^{*}$ and $\mathbf{e}_{2}^{*}$ with real number coefficients, as shown in Fig.1. If other two noncollinear reciprocal vectors are chosen as primitive reciprocal vectors, similar results will emerge as well. The distinct nonzero coefficients for these 12 vectors are $1,1 / 2$, and $\sqrt{3} / 2$. When applying CAM to this example of 12 -fold vectors, we need an integer $L$ such that

$$
\left|L \cdot\left(1, \frac{\sqrt{3}}{2}, \frac{1}{2}\right)-\left(L,\left[\frac{\sqrt{3}}{2} L\right],\left[\frac{1}{2} L\right]\right)\right|_{l^{\infty}} \rightarrow 0
$$




\section{Applying CAM to Lifshitz-Petrich Method}

In CAM, we indeed use crystalline approximants to approximate quasicrystals. By substituting Eqn (10) into (1), the Lifshitz-Petrich free energy density functional becomes

$$
\begin{aligned}
F[\phi]= & \frac{1}{2} \sum_{\mathbf{k}_{1}+\mathbf{k}_{2}=0}\left[c\left(1-|\mathbf{k}|^{2}\right)^{2}\left(q^{2}-|\mathbf{k}|^{2}\right)^{2}-\varepsilon\right] \hat{\phi}\left(\mathbf{k}_{1}\right) \hat{\phi}\left(\mathbf{k}_{2}\right) \\
& -\frac{\alpha}{3} \sum_{\mathbf{k}_{1}+\mathbf{k}_{2}+\mathbf{k}_{3}=0} \hat{\phi}\left(\mathbf{k}_{1}\right) \hat{\phi}\left(\mathbf{k}_{2}\right) \hat{\phi}\left(\mathbf{k}_{3}\right)+\frac{1}{4} \sum_{\mathbf{k}_{1}+\mathbf{k}_{2}+\mathbf{k}_{3}+\mathbf{k}_{4}=0} \hat{\phi}\left(\mathbf{k}_{1}\right) \hat{\phi}\left(\mathbf{k}_{2}\right) \hat{\phi}\left(\mathbf{k}_{3}\right) \hat{\phi}\left(\mathbf{k}_{4}\right) .
\end{aligned}
$$

Then we use the semi-implicit scheme (4) to minimize the free energy density functional. For CAM, the semi-implicit method becomes

$$
\left(\frac{1}{\Delta t}+c\left(1-\mathbf{k}^{2}\right)^{2}\left(q^{2}-\mathbf{k}^{2}\right)^{2}\right) \hat{\phi}_{t+\Delta t}(\mathbf{k})=\left(\frac{1}{\Delta t}+\varepsilon\right) \hat{\phi}_{t}(\mathbf{k})+\alpha\left(\phi_{t}^{2}\right)(\mathbf{k})-\left(\phi_{t}^{3}\right)(\mathbf{k}),
$$

where $\left(\phi_{t}^{m}\right)(\mathbf{k})=\int d \mathbf{r} \phi^{m}(\mathbf{r}) e^{-i \mathbf{k r}}, m=2,3$. The right terms of the dynamics can be

efficiently calculated by the pseudospectral method 30 . The Laplacian terms are computed in $d$-dimensional reciprocal space easily, while the convolutions can be calculated efficiently by Fast-Fourier transformation (FFT). Then the computational complexity is $O(N \log N)$ at each time step, $N$ is the number of degrees of freedom.

\section{B. Projection Method (PM)}

\section{Method Description of PM}

As mentioned above, CAM computes the crystalline approximants. However, the approximation method can not evaluate the free energy density exactly because of the approximate error of SDA. Therefore it is necessary to design a new numerical method that improves the calculation of quasicrystals by avoiding the approximate error of SDA. From the higher-dimensional description ${ }^{16}$, there exists an equivalent $n$-dimensional representation of a $d$-dimensional quasicrystal $(n>d)$. The reciprocal vectors $\mathbf{k} \in \mathbb{R}^{d}$ of a $d$-dimensional quasicrystal are

$$
\mathbf{k}=h_{1} \mathbf{p}_{1}^{*}+h_{2} \mathbf{p}_{2}^{*}+\cdots+h_{n} \mathbf{p}_{n}^{*}, \quad h_{i} \in \mathbb{Z},
$$

with vectors $\mathbf{p}_{i}^{*} \in \mathbb{R}^{d}$ of rank $n$. In order to develop the new numerical method, we firstly redefine the selected reciprocal vectors $\mathbf{p}_{i}^{*}$ with $n$ components. Then the quasicrystal can 
be represented by these redefined basic reciprocal vectors with integral coefficients in $n$ dimensional space. This representation has the advantage, for our purpose, that the $d$ dimensional quasicrystal is periodic in $n$-dimensions. Based on the redefined reciprocal vectors, we introduce the $n$-dimensional reciprocal lattice. Assume that $n$-dimensional vectors, $\mathbf{b}_{1}^{*}, \mathbf{b}_{2}^{*}, \ldots, \mathbf{b}_{n}^{*}$, are the primitive reciprocal vectors of a 1 st Brillouin zone in $n$-dimensional reciprocal space, the reciprocal vector of $n$-dimensional periodic structure can be expressed as

$$
\mathbf{H}=h_{1} \mathbf{b}_{1}^{*}+h_{2} \mathbf{b}_{2}^{*}+\cdots+h_{n} \mathbf{b}_{n}^{*}
$$

where the coefficient $h_{i} \in \mathbb{Z}$, and $\mathbf{H} \in \mathbb{R}^{n}$. The correspondingly primitive vectors of the direct space $\mathbf{b}_{i}$ satisfy the dual relationship (6). We want to use the $n$-dimensional reciprocal vector $\mathbf{H}$ to represent the $d$-dimensional quasicrystal. Then we can solve a $d$-dimensional quasicrystal as a periodic structure in $n$-dimensional space. The key point of implementing the above idea is to provide an operator to project the $n$-dimensional structure into $d$ dimensional space.

In order to solve the problem, we propose a novel Fourier expansion for the $d$-dimensional quasiperiodic function

$$
g(\mathbf{r})=\sum_{\mathbf{H}} \hat{g}(\mathbf{H}) e^{i\left[(\mathcal{S} \cdot \mathbf{H})^{T} \cdot \mathbf{r}\right]} .
$$

In the expression, $\mathbf{r} \in \mathbb{R}^{d}, \mathbf{H} \in \mathbb{R}^{n}$, and $\mathcal{S}$ is the projective matrix which connects the $d$-dimensional physical space with the $n$-dimensional reciprocal space. We note that the two representations (15) and (16) can be used to describe the same quasicrystal. The reciprocal vectors of a $d$-dimensional quasicrystal can be represented by $d$-dimensional reciprocal vectors $\mathbf{p}_{i}^{*}$ with integral coefficients, and also the integral combinations of extended $n$ dimensional primitive reciprocal vectors $\mathbf{b}_{i}^{*}$. Therefore, we can obtain the projective matrix $\mathcal{S}$ through projecting the $n$-dimensional reciprocal vectors $\mathbf{b}_{i}$ into $d$-dimensional reciprocal space, i.e., $\mathbf{p}_{i}=(\mathcal{S} \cdot \mathbf{b})_{i}, i=1,2, \ldots, n$. The $j$-th component $p_{i j}^{*}$ of the projected reciprocal vector $\mathbf{p}_{i}^{*}$ can be expressed by $\mathbf{b}_{i}^{*}$

$$
p_{i j}^{*}=\sum_{m=1}^{n} s_{j m} b_{i m}^{*}, \quad j=1,2, \ldots, d,
$$

where $b_{i m}^{*}$ is the $m$-th component of the $i$-th $n$-dimensional reciprocal vector $\mathbf{b}_{i}^{*}$. These coefficients $s_{j m} \in \mathbb{R}, j=1,2, \ldots, d, m=1,2, \ldots, n$, form the $d \times n$-order nonzero projective 
matrix $\mathcal{S}$ which reflects the symmetry of quasicrystals. For the least computational expense, the dimension $n$ of the extended space must be the smallest determined by the order of the elements of the symmetric group $\stackrel{16[17}{ }$. For example, 5-, 8-, 10-, and 12-fold symmetric quasicrystals, the dimension of embedded space is four. However, 7-, 9-, and 18-fold symmetric quasicrystals must be restricted to six-dimensional space. The projective matrix $\mathcal{S}$ is not unique, which is determined by the selection of reciprocal vectors $\mathbf{b}_{i}^{*}$. The represented coefficients of reciprocal vectors are dependent on the selection of primitive reciprocal vectors, however, the reciprocal vectors of a quasicrystal are unique. Therefore, the selection of basic reciprocal vectors $\mathbf{b}_{i}^{*}$ as well as the projective matrix $\mathcal{S}$ is irrelevant to the quasicrystal. Considering a periodic structure, the projective matrix $\mathcal{S}$ degenerates to a $d \times d$-order unit matrix.

Furthermore, PM can be extended to calculate one-dimensional quasicrystals even though the notion of rotational symmetry does not exist in one dimension. If an energy functional has one-dimensional quasiperiodic structures with different incommensurate scales, for example,

$$
g(x)=C_{0} \sin (x)+C_{1} \sin \left(q_{1} x\right)+C_{2} \sin \left(q_{2} x\right)+\cdots,
$$

where the common multiples of $1, q_{1}, q_{2}, \ldots$, are irrational numbers in pairs, the projective matrix becomes a vector

$$
\mathcal{S}=\left(1, q_{1}, q_{2}, \ldots\right)
$$

The present approach can be applied to calculate one-dimensional quasicrystals.

In the following, a lemma is given to indicate which variable should be computed in PM.

Lemma 1. For a d-dimensional quasiperiodic function $g(\mathbf{r})$, under the expansion (17), we have

$$
\lim _{V \rightarrow \infty} \frac{1}{V} \int d \mathbf{r} g(\mathbf{r})=\left.\hat{g}(\mathbf{H})\right|_{\mathbf{H}=0}
$$

Proof. Firstly, we note that

$$
\lim _{V \rightarrow \infty} \frac{1}{V} \int d \mathbf{r} \exp \{(\mathcal{S} \cdot \mathbf{H}) \cdot \mathbf{r}\}=\delta(\mathcal{S} \cdot \mathbf{H})
$$

Therefore,

$$
\lim _{V \rightarrow \infty} \frac{1}{V} \int d \mathbf{r} g(\mathbf{r})=\lim _{V \rightarrow \infty} \frac{1}{V} \int d \mathbf{r} \sum_{\mathbf{H}} \hat{g}(\mathbf{H}) e^{i\left[(\mathcal{S} \cdot \mathbf{H})^{T} \cdot \mathbf{r}\right]}=\left.\hat{g}(\mathbf{H})\right|_{\mathcal{S} \cdot \mathbf{H}=0} .
$$


Then we just need to prove that the expressions (21) and (23) are equivalent. From the definition of (16), $n$-dimensional reciprocal vector $\mathbf{H}$ is the integer-valued combinations of linearly independent primitive reciprocal vectors $\mathbf{b}_{i}^{*}, i=1,2, \ldots, n$. Because the projective matrix $\mathcal{S}$ is linear, the $d$-dimensional projected vector $\mathcal{S} \cdot \mathbf{H}$ is also the integer-valued combinations of the $d$-dimensional projected vectors $\mathbf{p}_{i}^{*}=\mathcal{S} \cdot \mathbf{b}_{i}^{*} \cdot \mathcal{S} \cdot \mathbf{H}=0$ is equivalent to the integral coefficients $h_{i}$ in Eqn. (23) to be zero. It means that the $n$-dimensional reciprocal vector $\mathbf{H}=0$.

Remark 1. From Lemma 1, the Fourier coefficients $\hat{g}(\mathbf{H})$ rather than $\hat{g}(\mathcal{S} \cdot \mathbf{H})$ should be computed in PM.

In the PM, a quasicrystal is computed in $n$-dimensional reciprocal space as a periodic structure, then the $n$-dimensional structure is projected into $d$-dimensional space to obtain the $d$-dimensional quasicrystal through projective matrix. Since different periodic phases have their own periodicity, the appropriate computational box is important to determine the final morphology of solutions, especially for complex phases. For example, in diblock copolymer systems $\frac{31}{31}$, the lamellae phase can be obtained easily in any computational region, however, for complex gyroid pattern, the computational box should be close to its period. For more complex quasicrystal, the computational box should be estimated carefully before computing. We also note that a equilibrium periodic structure is not only the minimum of a free energy density $F$ with respect to order parameters, but also with respect to the unit cell 30 . Therefore, the unit cell in $n$-dimensional space, $\mathcal{B}=\left[\mathbf{b}_{1}, \mathbf{b}_{2}, \ldots, \mathbf{b}_{n}\right]$, should satisfy $\partial F / \partial \mathcal{B}=0$. We will give a estimation formula for the unit cell of quasicrystals in the Lifshitz-Petrich model (see Sec. III B 3).

With the help of $n$-dimensional reciprocal space, the PM can calculate the spectrum of quasicrystals directly. In PM, the physical space variable $\mathbf{r}$ always belongs to $d$-dimensional space. Therefore an energy functional including $d$-dimensional quasicrystals should not be up to describing $n$-dimensional structures. The PM is able to compute quasicrystals rather than crystalline approximants. The PM is also using the periodic condition conveniently in higher-dimensional reciprocal space . Compared with CAM, the most important advantage of PM is that the method overcomes the restriction of SDA. Therefore PM can compute the free energy density to high accuracy numerically. Meanwhile, the proposed method can implement in a $n$-dimensional reciprocal unit cell to reduce computational cost. 


\section{An Example of 12-fold Rotational Symmetry}

As an example, we take the 12-fold rotational symmetry in two dimensions to illustrate the idea of PM. As Sec.IIIA2 discussed, the 12 reciprocal lattice vectors can not be represented

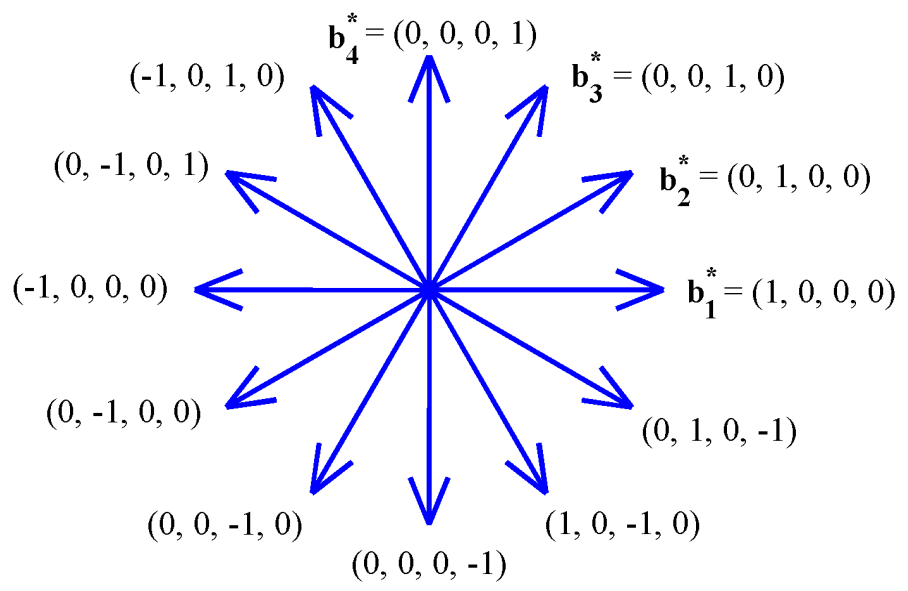

FIG. 2: Sets of reciprocal lattice vectors $\{\mathbf{H}\}$ of the 12-fold rotational symmetry with 4 components.

by two noncollinear vectors $\mathbf{e}_{1}^{*}$ and $\mathbf{e}_{2}^{*}$ with integral coefficients. However, as Fig.2 shows, if the vectors of $\mathbf{b}_{1}^{*}, \mathbf{b}_{2}^{*}, \mathbf{b}_{3}^{*}$, and $\mathbf{b}_{4}^{*}$ with 4 components are chosen as primitive reciprocal vectors, other reciprocal lattice vectors can be represented as integral combinations of these primitive vectors. The selected 4 vectors make up a basis in 4-dimensional space. The 2-dimensional 12-fold example is related to a periodic structure in 4-dimensional reciprocal space. The projected vectors $\mathbf{p}_{i}^{*}=\mathcal{S} \cdot \mathbf{b}_{i}^{*}, i=1,2,3,4$ also can be expressed by $\mathbf{e}_{1}^{*}$, $\mathbf{e}_{2}^{*}$ in 2-dimensional space, i.e.,

$$
p_{i 1}^{*}=\cos \frac{(i-1) \pi}{6} e_{11}^{*}, \quad p_{i 2}^{*}=\sin \frac{(i-1) \pi}{6} e_{22}^{*} .
$$

Therefore, the projective matrix is

$$
\mathcal{S}=\left(\begin{array}{llll}
1 & \cos (\pi / 6) & \cos (\pi / 3) & 0 \\
0 & \sin (\pi / 6) & \sin (\pi / 3) & 1
\end{array}\right)
$$




\section{Applying PM to Lifshitz-Petrich Method}

Applying PM to the Lifshitz-Petrich model in two dimensions, the order parameter $\phi(x, y)$ is expanded as

$$
\phi(x, y)=\sum_{\mathbf{H}} \hat{\phi}(\mathbf{H}) \exp \left\{i(\mathcal{S} \cdot \mathbf{H})^{T} \cdot(x, y)^{T}\right\}
$$

where $\mathbf{H}$ is a $n$-dimensional vector, $\mathcal{S}$ is a $2 \times n$-order projective matrix. For quasicrystals, the system volume $V$ should go to infinity. Based on Lemma 1, the Lifshitz-Petrich free energy density functional becomes

$$
\begin{aligned}
F[\phi(x, y)] & =\frac{1}{2} \sum_{\mathbf{H}_{1}+\mathbf{H}_{2}=0}\left\{c\left[1-\left(g_{1}^{2}+g_{2}^{2}\right)\right]^{2}\left[q^{2}-\left(g_{1}^{2}+g_{2}^{2}\right)\right]^{2}-\varepsilon\right\} \hat{\phi}\left(\mathbf{H}_{1}\right) \hat{\phi}\left(\mathbf{H}_{2}\right) \\
& -\frac{\alpha}{3} \sum_{\mathbf{H}_{1}+\mathbf{H}_{2}+\mathbf{H}_{3}=0} \hat{\phi}\left(\mathbf{H}_{1}\right) \hat{\phi}\left(\mathbf{H}_{2}\right) \hat{\phi}\left(\mathbf{H}_{3}\right)+\frac{1}{4} \sum_{\mathbf{H}_{1}+\mathbf{H}_{2}+\mathbf{H}_{3}+\mathbf{H}_{4}=0} \hat{\phi}\left(\mathbf{H}_{1}\right) \hat{\phi}\left(\mathbf{H}_{2}\right) \hat{\phi}\left(\mathbf{H}_{3}\right) \hat{\phi}\left(\mathbf{H}_{4}\right),
\end{aligned}
$$

where $g_{1}$ and $g_{2}$ are defined by

$$
\mathcal{S} \cdot \mathbf{H}=\left(\sum_{i=1}^{n} s_{1 i} \sum_{j=1}^{n} h_{j} b_{j i}, \sum_{i=1}^{n} s_{2 i} \sum_{j=1}^{n} h_{j} b_{j i}\right)^{T} \triangleq\left(g_{1}, g_{2}\right)^{T},
$$

$h_{j} \in \mathbb{Z}, s_{1 j}$ and $s_{2 j}$ are the components of the projective matrix $\mathcal{S}$, and $b_{j i}$ is the $i$-th component of the primitive reciprocal vector $\mathbf{b}_{j}$. The $n$-dimensional Fourier coefficient $\hat{\phi}(\mathbf{H})$ can be solved by the semi-implicit method (4)

$$
\begin{aligned}
\left(\frac{1}{\Delta t}+c\left(1-g_{1}^{2}\right.\right. & \left.\left.-g_{2}^{2}\right)^{2}\left(q^{2}-g_{1}^{2}-g_{2}^{2}\right)^{2}\right) \hat{\phi}_{t+\Delta t}(\mathbf{H}) \\
& =\left(\frac{1}{\Delta t}+\varepsilon\right) \hat{\phi}_{t}(\mathbf{H})+\alpha\left(\phi_{t}^{2}\right)(\mathbf{H})-\left(\phi_{t}^{3}\right)(\mathbf{H}),
\end{aligned}
$$

where the quadratic term and the third term are

$$
\left(\phi_{t}^{2}\right)(\mathbf{H})=\sum_{\mathbf{H}_{\mathbf{1}}+\mathbf{H}_{2}=\mathbf{H}} \hat{\phi}_{t}\left(\mathbf{H}_{1}\right) \hat{\phi}_{t}\left(\mathbf{H}_{2}\right), \quad\left(\phi_{t}^{3}\right)(\mathbf{H})=\sum_{\mathbf{H}_{\mathbf{1}}+\mathbf{H}_{2}+\mathbf{H}_{3}=\mathbf{H}} \hat{\phi}_{t}\left(\mathbf{H}_{1}\right) \hat{\phi}_{t}\left(\mathbf{H}_{2}\right) \hat{\phi}_{t}\left(\mathbf{H}_{3}\right) .
$$

In Eqn. (29), the linear terms can be solved easily. The nonlinear terms are $n$-dimensional convolutions in reciprocal space. Directly computing will result in expansive computational cost. However these convolutions are local point multiplication in $n$-dimensional direct space. Therefore we use the pseudospectral method ${ }^{30}$ to treat these terms by FFT. It should be emphasized that the PM is implemented in $n$-dimensional reciprocal space instead of in 
$d$-dimensional physical space. Computing these convolutions in $n$-dimensional direct space is to reduce computational complexity.

For PM, we give a method to estimate the $n$-dimensional unit cell for the Lifshitz-Petrich model ${ }^{31}$. Without loss of generality, we can choose a proper coordinate system such that $b_{i i} \neq 0, b_{i j}=0$, when $j>i$. If $\mathcal{B}=\left[\mathbf{b}_{1}, \mathbf{b}_{2}, \ldots, \mathbf{b}_{n}\right]$ is a $n$-dimensional unit cell of a $d$ dimensional quasicrystal. The first deviations of the free energy functional with respect to $b_{i j}$ should be zero, where $i=1,2, \ldots n, j=1,2, \ldots, i$, i.e.,

$$
\sum_{\mathbf{H}} h_{j}\left(g_{1} s_{1 i}+g_{2} s_{2 i}\right)\left[1-\left(g_{1}^{2}+g_{2}^{2}\right)\right]\left[q^{2}-\left(g_{1}^{2}+g_{2}^{2}\right)\right]\left[1+q^{2}-2\left(g_{1}^{2}+g_{2}^{2}\right)\right] \cdot|\hat{\phi}(\mathbf{H})|^{2}=0 .
$$

Then the unit cell can be obtained by solving the Eqn. (31). Since the Fourier coefficients $\phi_{\mathbf{H}}$ are unknown beforehand. In practice, only primary reciprocal vectors with equal Fourier coefficients because of symmetries are considered in estimating the unit cell. Therefore the Fourier coefficients $\hat{\phi}(\boldsymbol{H})$ in (31) will be cancelled and make no impression on evaluating the size of box.

\section{Computational Complexity}

In this section, we will give a general analysis on the computational complexity of CAM and PM in solving the two-dimensional Lifshitz-Petrich model. The computational complexity of CAM is dependent on the approximate accuracy of SDA (11) and the numerical precision. However, the PM overcomes the restriction of SDA, whose computational complexity is only dependent on the numerical accuracy. As described in Sec III A 1 and Sec. IIIB 1, both CAM and PM can use pseudospectral method in computing the two-dimensional LifshitzPetrich model. The number of basic functions used in the reciprocal space can be equivalent to the number of discretized points in the direct space. In order to guarantee the same numerical precision, we assume that the mesh step size is $\Delta x$ both for CAM (2 dimensions) and PM ( $n$ dimensions) in the direct space.

In the Lifshitz-Petrich model, the CAM is implemented in two dimensions. At a desired approximate error $E_{S D A}$ as well as the integer $L$, the number of the plane waves is $N_{\mathbf{k}} \times N_{\mathbf{k}}$,

$$
N_{\mathbf{k}}=\left[\frac{D_{\mathbf{k}}}{\Delta x}\right], \quad D_{\mathbf{k}}=2 \pi \times L
$$


where $[\cdot]$ rounds the number $\cdot$ to the nearest integer. The computational complexity of CAM

$$
O\left(N_{t, \mathbf{k}} \cdot 2 N_{\mathbf{k}}^{2} \log N_{\mathbf{k}}\right),
$$

where $N_{t, \mathbf{k}}$ is the number of time iterations.

In PM, the 2-dimensional quasicrystal is represented in $n$-dimensional reciprocal space as a periodic structure. The dimension of $n$ is dependent on the rotational symmetry of a quasicrystal. Therefore the 2-dimensional quasicrystal can be computed in a $n$-dimensional unit cell. We assume that the size of the $n$-dimensional unit cell is $D_{\mathbf{H}}$ in the direct space. The number of the plane-wave functions is $N_{\mathbf{H}}^{n}$, with

$$
N_{\mathbf{H}}=\left[\frac{D_{\mathbf{H}}}{\Delta x}\right] .
$$

As discussed in Sec.IIIB 1, the pseudospectral method in PM is used to solve the dynamical equation 29) in $n$-dimensional reciprocal space with the help of FFT. Therefore the computational complexity of PM is

$$
O\left(N_{t, \mathbf{H}} \cdot n N_{\mathbf{H}}^{n} \log N_{\mathbf{H}}\right),
$$

where $N_{t, \mathbf{H}}$ is the number of time iterations.

From the estimation formulas (33), the computational complexity of CAM is a function of the integer $L$, which is dependent upon the approximate error of SDA. In next section, we will find that $D_{\mathbf{k}} \gg D_{\mathbf{H}}$ as the approximate error of SDA decreases. The computational complexity of CAM may be larger than that of PM in a situation where higher numerical accuracy is required. More importantly, PM can compute quasicrystals and their free energy density to high accuracy without any approximate error of SDA. On the contrary, CAM always has the approximate error of SDA unless the computational box goes to infinity which results in unaccepted computational cost.

\section{NUMERICAL RESULTS AND DISCUSSION}

We will demonstrate the behavior of the two numerical methods, CAM and PM, based

on the two-dimensional Lifshitz-Petrich model. In previous research ${ }^{26}$, the Lifshitz-Petrich model showed that if $q$ is chosen around $2 \cos (\pi / 12)$ one can obtain a 2-dimensional quasicrystal with dodecagonal (12-fold) symmetry. The earlier work found that no choice of $q$ 
yields globally stable octagonal or decagonal symmetric pattern. However, recent study 29 manifests that the decagonal quasicrystal is stable in the Lifshitz-Petrich model. In this work we just consider the dodecagonal symmetric structure.

\section{A. Computational Complexity of Dodecagonal Symmetric Structure}

For the dodecagonal quasicrystal (DDQC), the initial reciprocal vectors at which the Fourier coefficients are nonzero, are shown in Fig.3. They contain two 12-fold stars of wave vectors, one on $|\mathbf{k}|=1$ and other on $|\mathbf{k}|=q=2 \cos (\pi / 12)^{26}$. The specific reciprocal

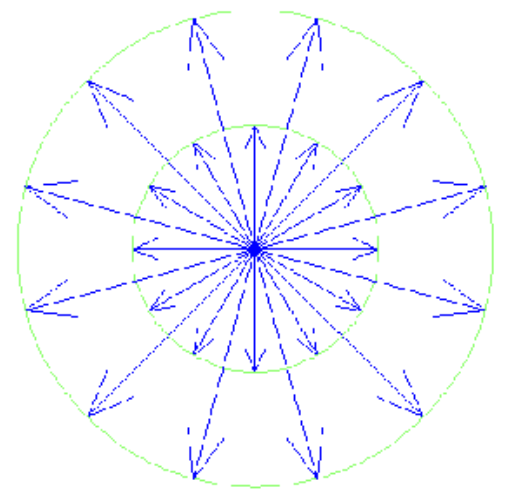

FIG. 3: Initial reciprocal lattice vectors for DDQC.

TABLE I: Initial reciprocal lattice vectors for DDQC in 2-dimensional space with

$$
q=2 \cos (\pi / 12) \text {. }
$$

$$
\begin{array}{|l|c|}
\hline|\mathbf{k}|=1 & (\cos (j \pi / 6), \sin (j \pi / 6)), j=0,1, \ldots, 11 \\
\hline|\mathbf{k}|=q & (q \cos (j \pi / 6+\pi / 12), q \sin (j \pi / 6+\pi / 12)), j=0,1, \ldots, 11 \\
\hline
\end{array}
$$

vectors are given in Tab. [1] when $\mathbf{e}_{1}^{*}=(1,0)$ and $\mathbf{e}_{2}^{*}=(0,1)$, as shown in Fig[1, are chosen as the basic reciprocal vectors in 2 dimensions. The distinct nonzero represented coefficients of these initial reciprocal vectors for DDQC phase are $1,1 / 2, \sqrt{3} / 2,2 \cos (\pi / 12), \cos (\pi / 12)$ and $\sqrt{3} \cos (\pi / 12)$. The condition of SDA 11 must be satisfied when one uses CAM. Fig. 4 shows the trend of the approximate error of SDA, $E_{S D A}$, as the integer $L$ increases. The convergence of $E_{S D A}$ is not uniform. Tab. [II gives the minimal integers for desired approximate error 


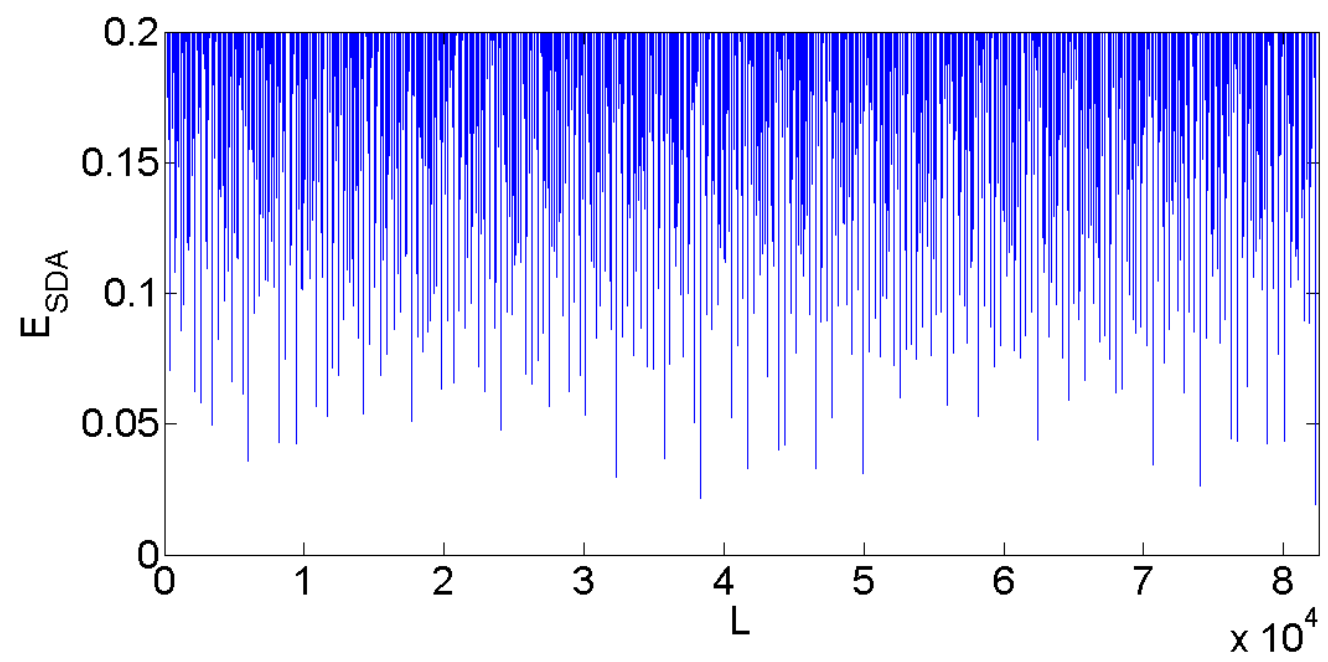

FIG. 4: The trend of the approximate error of SDA, $E_{S D A}$, as a function of the integer $L$.

TABLE II: The minimal integer $L$ for desired approximate error of SDA, $E_{S D A}$.

\begin{tabular}{|c|c|c|c|c|c|c|c|}
\hline$E_{S D A}$ & 0.19098 & 0.17486 & 0.07042 & 0.04953 & 0.03583 & 0.02961 & 0.01936 \\
\hline$L$ & 30 & 208 & 410 & 3404 & 6016 & 32312 & 82262 \\
\hline
\end{tabular}

The size of computational box used in CAM is $D_{\mathbf{k}}=2 \pi L$.

$E_{S D A}$, and the corresponding size $D_{\mathbf{k}}$ of computational box used in CAM. Then the initial reciprocal vectors in CAM are $[L \cdot \mathbf{k}]$. From the table, we find that $L$ increases quickly as the approximate error becomes small. Accordingly, the computational cost will increase greatly. We also find the $E_{S D A} \approx 0.19098(L=30)$ is the least requirement for computing dodecagonal symmetric structure, it is consistent with the result in Ref. 26. The initial reciprocal vectors in 4-dimensional space representation of PM are shown in Tab.III when the primitive reciprocal vectors are $\mathbf{b}_{1}^{*}=(1,0,0,0), \mathbf{b}_{2}^{*}=(0,1,0,0), \mathbf{b}_{3}^{*}=(0,0,1,0)$, and $\mathbf{b}_{4}^{*}=(0,0,0,1)$, as shown in Fig 2 . We can use the 24 initial reciprocal vectors in Tab. III to

TABLE III: Initial reciprocal lattice vectors $\{\mathbf{H}\}$ for DDQC in PM with $q=2 \cos (\pi / 12)$.

\begin{tabular}{|c|c|}
\hline$|\mathcal{S} \cdot \mathbf{H}|=$ & $\begin{array}{l}\left(\begin{array}{llll}0 & 1 & 0 & -1\end{array}\right)\left(\begin{array}{llll}0 & -1 & 0 & 1\end{array}\right)\left(\begin{array}{llll}1 & 0 & 0 & 0\end{array}\right)\left(\begin{array}{llll}-1 & 0 & 0 & 0\end{array}\right)\left(\begin{array}{llll}0 & 1 & 0 & 0\end{array}\right)\left(\begin{array}{llll}0 & -1 & 0 & 0\end{array}\right) \\
\left(\begin{array}{llll}0 & 0 & 1 & 0\end{array}\right)\left(\begin{array}{llll}0 & 0 & -1 & 0\end{array}\right)\left(\begin{array}{llll}0 & 0 & 0 & 1\end{array}\right)\left(\begin{array}{lllll}0 & 0 & 0 & -1\end{array}\right)\left(\begin{array}{llll}-1 & 0 & 1 & 0\end{array}\right)\left(\begin{array}{llll}1 & 0 & -1 & 0\end{array}\right)\end{array}$ \\
\hline$|\mathcal{S} \cdot \mathbf{H}|=$ & 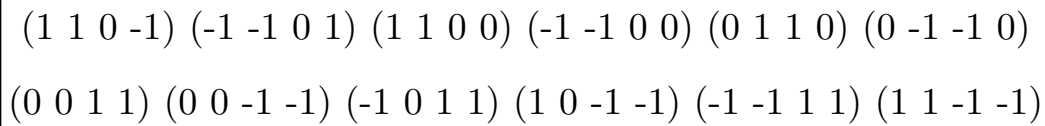 \\
\hline
\end{tabular}

The projective matrix $\mathcal{S}$ is given by Eqn. (25). 
estimate the unit cell of DDQC in 4-dimensional space. From the estimation formula 31 and the dual relationship (6), the size of the unit cell in $n$-dimensional direct space is $D_{\mathbf{H}}=2 \pi$. The projective matrix $\mathcal{S}$ of PM is given by Eqn. (25).

In the following, we will compare the computational complexity between CAM of different unit cell with $D_{\mathbf{k}}=2 \pi \cdot L$ and PM as discussed in Sec.IIIC. In each time step, for the dodecagonal symmetric structure, the computational complexity of CAM is

$$
C_{\mathbf{k}}=O\left(2 N_{\mathbf{k}}^{2} \log N_{\mathbf{k}}\right)=O\left(2\left(\frac{2 \pi L}{\Delta x}\right)^{2} \log \left(\frac{2 \pi L}{\Delta x}\right)\right)
$$

and the computational complexity of PM is

$$
C_{\mathbf{H}}=O\left(4 N_{\mathbf{H}}^{4} \log N_{\mathbf{H}}\right)=O\left(4\left(\frac{2 \pi}{\Delta x}\right)^{4} \log \left(\frac{2 \pi}{\Delta x}\right)\right) .
$$

For $L=30$ with $E_{S D A} \approx 0.19098$ in CAM, the computational complexity $C_{\mathbf{k}}<C_{\mathbf{H}}$ when $\Delta x$ is smaller than 0.20943951. However, for $L=208, C_{\mathbf{k}}<C_{\mathbf{H}}$ only if $\Delta x>500.6549$, which is much larger than the size of the unit cell $D_{\mathbf{H}}$. It can not be implemented numerically. The computational complexity $C_{\mathbf{H}}$ of $\mathrm{PM}$ is always smaller than that of $C_{\mathbf{k}}$. For higher approximate accuracy of SDA with larger $L, C_{\mathbf{H}}<C_{\mathbf{k}}$, and total computational complexity of PM may be less than CAM.

Subsequently we compare the total computational complexity including time iterations of CAM (with $L=30$ ) and PM through numerical experiments. All the methods considered here have been implemented in $\mathrm{C}$ language. Fourier transforms are computed using the FFTW ${ }^{32}$ package. The codes were run in the same workstation, a Intel(R) Xeon(R) CPU E5450 @3.00GH memory $16 \mathrm{G}$ under linux. The time step size, $\Delta t$, is always selected as 0.1 for both methods. To measure error we use $l^{\infty}$ norm,

$$
E_{C A M}=\max _{\mathbf{k}}\left\{\left(\frac{\delta F}{\delta \phi}\right)(\mathbf{k})\right\}
$$

for CAM, and

$$
E_{P M}=\max _{\mathbf{H}}\left\{\left(\frac{\delta F}{\delta \phi}\right)(\mathbf{H})\right\}
$$

for PM.

Fig. 5 shows the total CPU time reaching an error of $10^{-8}$ by CAM $(L=30)$ and PM at a set of parameters, $c=50, \varepsilon=0.015, \alpha=1.0$, with different discrete resolution. The 


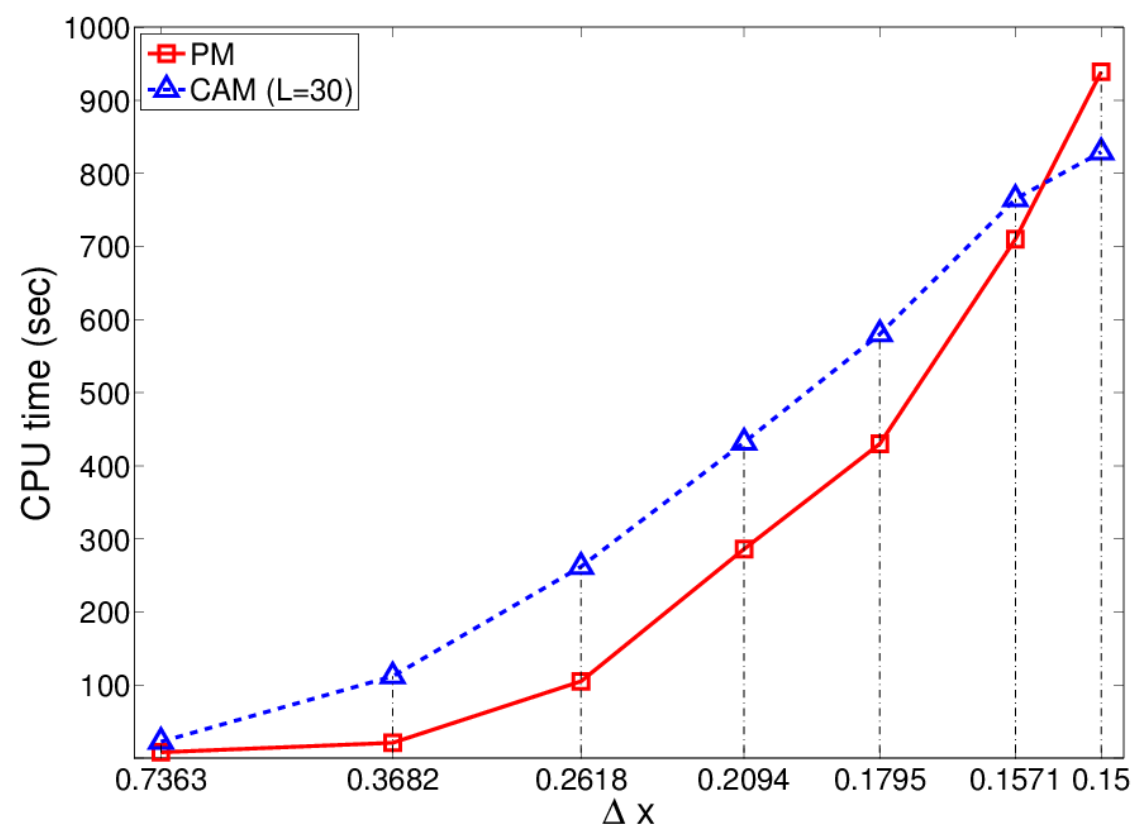

FIG. 5: The total CPU time required by CAM $(L=30)$ and PM at the error of $10^{-8}$, with different mesh step size $\Delta x$ at a set of parameters: $c=50, \varepsilon=0.015, \alpha=1.0$.

number of iterations on different grids is same. For CAM method, $N_{t, \mathbf{k}}=848$, while for PM approach, $N_{t, \mathbf{k}}=472$. It may be that the accuracy of spacial discretization is enough for the calculated structure at the set of parameter. For the same reason, the free energy density calculated by PM is nearly the same on these grids, $F_{P M}=-3.524067379 \mathrm{e}-03$, while the CAM obtains the equal free energy density $F_{C A M}=-3.457837609 \mathrm{e}-03$. However, the iterations of CAM, $N_{t, \mathbf{k}}$ is alway large than that of PM, $N_{t, \mathbf{H}}$. As discussed in Sec.IV B, it may be that PM keeps the rotational symmetry, while CAM dose not. Therefore PM can reach the same accuracy more quickly. The free energy density computed by PM is lower than the CAM. It may result from the approximate error of SDA in CAM (also see Fig 7(a)). As Fig 5 demonstrates, the cost of CPU time of PM is lower than that of CAM until the mesh step size $\Delta x$ is less than about 0.15. It is different from the above analysis about the computational complexity in each time step because of the different iterations between PM and CAM.

From these results, we find that with relative large mesh step size $\Delta x, \mathrm{PM}$ can obtain enough numerical accuracy with less computational cost than CAM $(L=30)$. CAM always has approximant error unless $D_{\mathbf{k}} \rightarrow \infty$. And as discussed in the section, higher accuracy 
of approximant error will result in heavy computational burder. Therefore, PM has less computational complexity than CAM to high accuracy in computing these quasicrystals represented in 4 dimensions, such as dodecagonal symmetric structures.

\section{B. Dodecagonal Symmetric Structure}

The initial reciprocal coefficients, as mentioned in Sec.IVA, at which the Fourier coefficients are nonzero are used as initial values to find equilibrium dodecagonal symmetric structures. The simulations of CAM are performed on a $256 \times 256$ grids with $L=30$, and the simulations of PM are performed with $N_{\mathbf{H}}^{4}=32^{4}$ plane waves. The projective matrix $\mathcal{S}$ of PM is given by expression (25). We also find that the morphologies and the free energy density will not change with denser grids. The morphology of the dodecagonal approximant computed by CAM in physical space is given in Fig.6(a), It is a periodic structure. The DDQC calculated by PM is shown in Fig.6(b). Both morphologies demonstrate the 12-fold

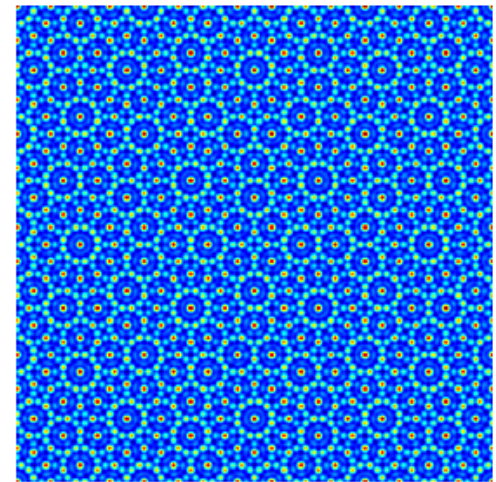

(a)Dodecagonal crystalline approximant

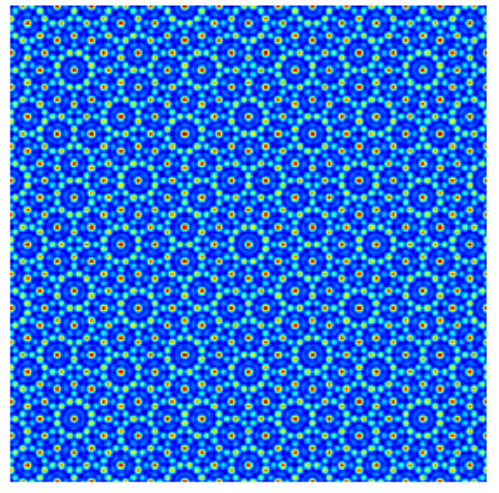

(b)DDQC

FIG. 6: The morphologies of (a). Dodecagonal approximant computed by CAM and (b). DDQC calculated by PM at $\varepsilon=0.015, \alpha=1, c=50, q=2 \cos (\pi / 12)$.

orientational symmetry in the physical space, at least locally. However, further analysis about the relationship of the Fourier coefficients will come to a different conclusion. From the rotational symmetry, each 12 reciprocal vectors with dodecagonal rotational symmetry on the ring $|\mathbf{k}|=1(|\mathcal{S} \cdot \mathbf{H}|=1)$, or $|\mathbf{k}|=q(|\mathcal{S} \cdot \mathbf{H}|=q)$, should have equal Fourier coefficients. Tab.IV shows the principle Fourier coefficients calculated by CAM. Tab.V gives 
these Fourier coefficients on principle reciprocal vectors calculated by PM. From these results, we can find the 12 Fourier coefficients calculated by CAM on each characteristic ring are not equal. In contrast, as Tab. $\mathrm{V}$ shown, PM can obtain the 12 equal Fourier coefficients

TABLE IV: The Fourier coefficients of the principle reciprocal vectors for dodecagonal crystalline approximant computed by CAM with $L=30$ at $\varepsilon=0.015, \alpha=1, c=150$,

\begin{tabular}{|c|c|c|}
\hline \multicolumn{1}{|c|}{$q=2 \cos (\pi / 12)}$. \\
\hline$|\mathbf{k}|=L$ & $\begin{array}{l}(30,0)(-30,0)(0,30)(0,-30) \\
(26,15)(-26,-15)(15,26)(-15,-26) \\
(-15,26)(15,-26)(-26,15)(26,-15)\end{array}$ & $\begin{array}{l}6.106618210 \mathrm{e}-02 \\
5.928204525 \mathrm{e}-02\end{array}$ \\
\hline$|\mathbf{k}|=[L \cdot q]$ & $\begin{array}{l}(41,41)(-41,-41)(-41,41)(41,-41) \\
(56,15)(-56,-15)(15,56)(-15,-56)\end{array}$ & $5.458000478 \mathrm{e}-02$ \\
\hline $\begin{array}{l}(-15,56)(15,-56)(-56,15)(56,-15) \\
5.683018766 \mathrm{e}-02\end{array}$ & - \\
\hline
\end{tabular}

on each ring. We also find the same phenomenon at different parameter coordinates. For

TABLE V: The Fourier coefficients of the principle reciprocal vectors for DDQC computed by PM at $\varepsilon=0.015, \alpha=1, c=150, q=2 \cos (\pi / 12)$.

\begin{tabular}{|c|c|c|}
\hline$|\mathcal{S} \cdot \mathbf{H}|=1$ & 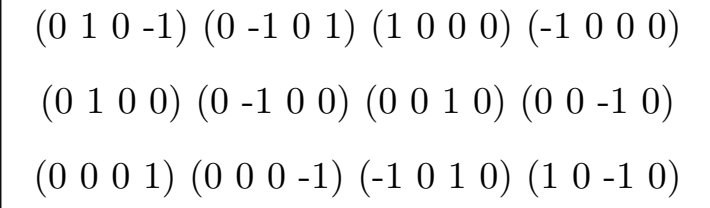 & $5.856822141 \mathrm{e}-02$ \\
\hline$|\mathcal{S} \cdot \mathbf{H}|=q$ & $\begin{array}{l}\left(\begin{array}{llll}1 & 1 & 0 & -1\end{array}\right)\left(\begin{array}{llll}-1 & -1 & 0 & 1\end{array}\right)\left(\begin{array}{llll}1 & 1 & 0 & 0\end{array}\right)\left(\begin{array}{llll}-1 & -1 & 0 & 0\end{array}\right) \\
\left(\begin{array}{llll}0 & 1 & 1 & 0\end{array}\right)\left(\begin{array}{llll}0 & -1 & -1 & 0\end{array}\right)\left(\begin{array}{llll}0 & 0 & 1 & 1\end{array}\right)\left(\begin{array}{llll}0 & 0 & -1 & -1\end{array}\right) \\
\left(\begin{array}{llll}-1 & 0 & 1 & 1\end{array}\right)\left(\begin{array}{llll}1 & 0 & -1 & -1\end{array}\right)\left(\begin{array}{llll}-1 & -1 & 1 & 1\end{array}\right)\left(\begin{array}{llll}1 & 1 & -1 & -1\end{array}\right)\end{array}$ & $5.855442187 \mathrm{e}-02$ \\
\hline
\end{tabular}

CAM, we also use higher approximate accuracy $E_{S D A} \approx 0.07042$, with the computational box $D_{\mathbf{k}} \times D_{\mathbf{k}}, D_{\mathbf{k}}=820 \pi$. The numerical experiments are implemented on a $4096 \times 4096$ grids. We find that CAM can capture the dodecagonal approximant, however, it still can not obtain 12 equal Fourier coefficients on each ring as well as the above numerical experiments. Therefore, we come to a conclusion that PM can keep the non-crystallographic symmetry to high accuracy, while CAM can not unless $D_{\mathbf{k}} \rightarrow \infty$. 


\section{Free Energy Density}

In this section, we compare the free energy density computed by CAM, PM and singlewave approximation (SWA) approaches. The SWA method uses principal Fourier vectors to calculate the free energy functional analytically under some constraints 33 . It translates the energy functional into a single-variable or multi-variable function. The minimum of free energy can be approximately obtained by minimizing the reduced energy function with respect to these variables. In the Lifshitz-Petrich model, the principal Fourier vectors of DDQC have been shown in Fig 3 . When $c \rightarrow \infty$, the reduced free energy function of DDQC pattern computed by SWA $\stackrel{26] 29]}{\text { is }}$

$F_{12}=99\left(\phi_{1}^{4}+\phi_{q}^{4}\right)+144\left(\phi_{1}^{3}+\phi_{1}^{3} \phi_{q}\right)+360 \phi_{1}^{2} \phi_{q}^{2}-24\left(\phi_{1}^{2} \phi_{q}+\phi_{1}^{2} \phi_{q}\right)-8\left(\phi_{1}^{3}+\phi_{q}^{3}\right)-6 \varepsilon\left(\phi_{1}^{2}+\phi_{q}^{2}\right) / \alpha^{2}$,

$\phi_{1}, \phi_{q} \in \mathbb{R}$ stand for Fourier coefficients on the $|\mathbf{k}|=1$ and $|\mathbf{k}|=q$ rings, respectively. The approximated minimum of DDQC can be obtained by minimizing the above equation with respect to $\phi_{1}$ and $\phi_{q}$.

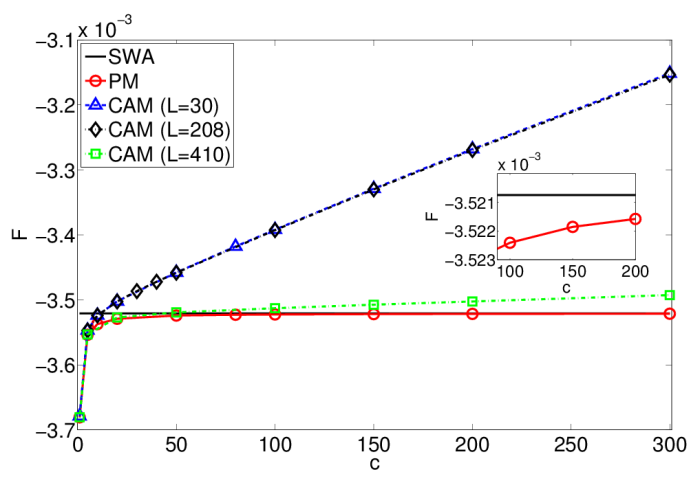

(a)Free energy density

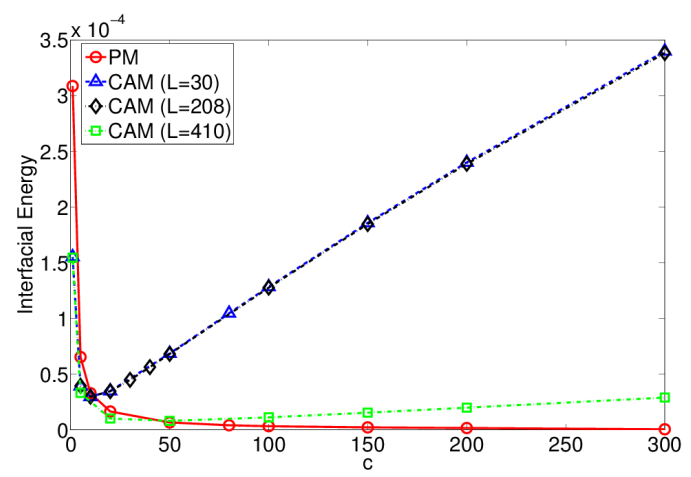

(b)Interfacial energy (Laplacian terms)

FIG. 7: (a). Free energy density calculated by PM and CAM, as a function of the penalty factor $c$, relative to that of SWA $(c \rightarrow \infty)$, with $q=2 \cos (\pi / 12), \alpha=1.0, \varepsilon=0.015$. (b).

Corresponding interfacial energy computed by PM and CAM.

Fig.7(a) shows the free energy density calculated by PM and CAM $(L=30,208,410)$, as a function of the penalty factor $c$, relative to that of SWA $(c \rightarrow \infty)$ with $q=2 \cos (\pi / 12)$, $\alpha=1.0, \varepsilon=0.015$. In numerical simulations, CAM are performed with $720 \times 720$ plan waves $(\Delta x=0.2618)$ for $L=30$; with $2048 \times 2048$ plan waves for $L=208$; with $4096 \times 4096$ 
TABLE VI: The CPU time required for minimizing the free energy (1) by the PM and CAM approaches at error of $10^{-6}$ with $q=2 \cos (\pi / 12), \alpha=1.0 \varepsilon=0.015$ for different $c$. In simulations, $\Delta t=0.1 ; N_{\boldsymbol{H}}=24$ utilized in PM; $N_{\boldsymbol{k}}=720,2048,4096$, used in CAM corresponding to $L=30,208,410$, respectively.

\begin{tabular}{|c||c|c|c|c|c|c|c|}
\hline \multicolumn{1}{|c||}{} & \multicolumn{7}{c|}{ CPU time (sec) } \\
\hline \hline$c$ & 5 & 20 & 50 & 100 & 150 & 200 & 300 \\
\hline $\mathrm{PM}$ & 45.86 & 40.91 & 40.38 & 39.49 & 39.69 & 39.72 & 39.39 \\
\hline $\mathrm{CAM}(L=30)$ & 122.49 & 136.97 & 143.10 & 181.89 & 189.29 & 185.33 & 197.05 \\
\hline $\mathrm{CAM}(L=208)$ & 1172.56 & 1381.57 & 1567.82 & 1716.40 & 1764.55 & 1896.65 & 1847.07 \\
\hline $\mathrm{CAM}(L=410)$ & 3457.19 & 3655.40 & 3397.97 & 3803.35 & 4035.10 & 4471.93 & 4767.31 \\
\hline
\end{tabular}

plan waves for $L=410.32^{4}(\Delta x=0.2618)$ plan waves were utilized in PM approach. The free energy density computed by CAM is heavily dependent on the approximate error of SDA. As Tab. II shown, when $L=30$ and 208, the approximate errors of SDA are 0.19098 and 0.17486. Under the approximate error, the CAM manifests the nearly same behavior. The free energy density $F_{C A M}$ computed by CAM is less than $F_{S W A}$ obtained by SWA until the penalty factor $c$ increases to about 10 . Then $F_{C A M}$ is larger than $F_{S W A}$ as $c$ increases. The reason is that the CAM can not control the principal reciprocal vectors located on $|\mathbf{k}|=1$ and $|\mathbf{k}|=q$ when the penalty factor $c$ increases, as shown in Fig.7(b), In other words, CAM can not calculate the interfacial energy (Laplacian terms) accurately when $c$ is large. However the role of the differential terms in the Lifshitz-Petrich model is to keep the interactions at two characteristic scales. It is one of the reasons that the Lifshitz-Petrich model is able to stabilize quasicrystals $\frac{26}{26}$. Correspondingly, the $F_{C A M}$ diverges from the true free energy density. We have also used $L=410$ which improves the approximate accuracy of SDA to $E_{S D A}=0.07042$ in CAM to observe the free energy density. It improves the accuracy of free energy, however, the same problem appeared when $c$ is larger than 50 . In contrast, the free energy density $F_{P M}$ calculated by PM is always smaller than $F_{S W A}$ for all $c$ and converges to the $F_{S W A}$ as $c \rightarrow \infty$. It is because the more basic functions are used in simulations by PM than that of SWA. The approximated space of PM is more precise than that of SWA. The PM can also maintain its principle reciprocal vectors located on scales 1 and $q$ which is consistent with the model, as shown in Fig.7(b), The corresponding 
CPU times spent in simulations are shown in Tab.VI. Compared with the CAM method, the PM can evaluate the free energy density to high numerical accuracy with economical computational cost in dodecagonal symmetric phase.

\section{CONCLUSIONS AND OUTLOOK}

In the article, we summary the features of CAM approach, and point out the advantage of using the periodic condition and the restriction condition of the SDA in this method. The errors of CAM come from two parts: the approximate error of SDA and numerical discretization. Subsequently we propose a new numerical method, the PM, with the help of higher-dimensional reciprocal space. The developed method overcomes the restriction of SDA and enables us view a quasicrystal as a periodic structure, and uses the periodic condition conveniently. The projection method can reduce the computational effort efficiently by computed quasicrystals in a higher-dimensional unit cell and using the pseudospectral method. By applying two methods to the Lifshitz-Petrich model, we analyze the computational complexity. The computational complexity of CAM is dependent on the approximate error and the numerical resolution, while that of PM is only dependent upon the numerical resolution. Specially, in computing dodecagonal symmetric pattern, PM has less computational complexity than that of CAM. We also find that our approach can keep the rotational symmetry accurately, and more significantly, the present algorithm can calculate the free energy density to high accuracy without any approximate error of SDA. However, the dimensions of our computational space are dependent on the rotational symmetry of quasicrystals. For quasiperiodic structures with 5-, 8-, 10- and 12-fold symmetry, the computational space dimension is four. PM has accepted computational complexity. For a quasicrystal with of 7-, 9-, and 18-fold quasicrystals, the dimension of computational space is up to six. It is also emphasized that the recently discovered quasicrystals can be all represented as periodic structures in less than 6-dimensional space. Our future work will focus on the improvement of the computational efficiency of the projection method, and make it to 6-dimensional cases. Finally, we should point out that PM can be also applied to study general $d$-dimensional

aperiodic structures $\frac{15}{15}$ whose Fourier spectrum consists of $\delta$-peaks, $\mathbf{H}=\sum_{i=1}^{n} h_{i} \mathbf{b}_{i}^{*}, h_{i} \in \mathbb{Z}$, of rank $n(n>d)$ with basis vectors $\mathbf{b}_{i}^{*}, i=1,2, \ldots, n$. 


\section{ACKNOWLEDGMENTS}

The authors would like to acknowledge Dr. Robert A. Wickham for his help with the English grammar and useful advice. The work is supported by the National Science Foundation of China 21274005 and 50930003, and the China Postdoctoral Science Foundation $2011 \mathrm{M} 500179$.

\section{REFERENCES}

${ }^{1}$ D. Shechtman, I. Blech, D. Gratias, and J.W. Cahn, Metallic phase with long-range orientational order and no translational symmetry, Phys. Rev. Lett. 53 (1984) 1951.

${ }^{2}$ D. Levine and P. J. Steinhardt, Quasicrystals: a new class of ordered structures, Phys. Rev. Lett. 53 (1984) 2477.

${ }^{3}$ R. Penrose, The role of aesthetics in pure and applied mathematical research, Inst. Math. Appl. 10 (1974) 266.

${ }^{4}$ M. Gardner, Extraordinary nonperiodic tiling that enriches the theory of tiles, Sci. Am. 236 (1977) 110.

${ }^{5} \mathrm{~W}$. Steurer, Twenty years of structure research on quasicrystals. part I. pentagonal, octagonal, decagonal and dodecagonal quasicrystals, Z. Krist, 219 (2004) 391.

${ }^{6}$ A. P. Tsai, Icosahedral clusters, icosaheral order and stability of quasicrystals-view of metallurgy, Sci. Technol. Adv. Mat. 9 (2008) 013008, and references therein.

${ }^{7}$ X. Zeng, G. Ungar, Y. Liu, V. Percec, A. E. Dulcey, and J. K. Hobbs, Supramolecular dendritic liquid quasicrystals, Nature 428 (2004) 157.

${ }^{8}$ K. Hayashida, T. Dotera, A. Takano, and Y. Matsushita, Polymeric quasicrystal: Mesoscopic quasicrystalline tiling in ABC star polymers, Phys. Rev. Lett. 98 (2007) 195502.

${ }^{9}$ D. V. Talapin, E. V. Shevchenko, M. I. Bodnarchuk, X. Ye, J. Chen, and C. B. Murray, Quasicrystalline order in self-assembled binary nanoparticle superlattices, Nature 461 (2009) 964.

${ }^{10}$ S. Fischer, A. Exner, K. Zielske, J. Perlich, S. Deloudi, W. Steurer, P. Lindner, and S. Förster, Colloidal quasicrystals with 12-fold and 18-fold diffraction symmetry, P. Natl. Acad. Sci. USA 108 (2011) 1810. 
${ }^{11}$ G. Gompper and M. Schick, in Self-Assembling Amphiphilic Systems, edited by C. Domb and J. L. Lebowitz, Phase Transitions \& Critical Phenomena, Academic Press, London, 1994.

${ }^{12}$ T. Dotera, Mean-field theory of archimedean and quasicrystalline tilings, Philos. Mag. 87 (2007) 3011.

${ }^{13}$ K. Barkan, H. Diamant, and R. Lifshitz, Stability of quasicrystals composed of soft isotropic particles, Phys. Rev. B 83 (2011) 172201.

${ }^{14}$ A. I. Goldman and R. F. Kelton, Quasicrystals and crystalline approximants, Rev. Mod. Phys. 65 (1993) 213.

${ }^{15}$ T. Janssen, G. Chapuis, and M. De Boissieu, Aperiodic Crystals: From Modulated Phases to Quasicrystals, Oxford University Press, USA, 2007.

${ }^{16}$ W. Steurer and S. Deloudi, Crystallography of Quasicrystals: Concepts, Methods and Structures, Springer Verlag, 2009.

${ }^{17} \mathrm{H}$. Heller, The crystallographic restriction in higher dimensions, Acta Cryst. A 41, (1985) 541.

${ }^{18}$ D. A. Rabson, N. D. Mermin, D. S. Rokhsar, and D. C. Wright, The space groups of axial crystals and quasicrystals, Rev. Mod. Phys. 63 (1991) 699.

${ }^{19}$ N. D. Mermin, The space groups of icosahedral quasicrystals and cubic, orthorhombic, monoclinic, and triclinic crystals, Rev. Mod. Phys. 64 (1992) 3.

${ }^{20}$ J. Dräger and N. D. Mermin, Superspace groups without the embedding: the link between superspace and Fourier-space crystallography, Phys. Rev. Lett. 76 (1996) 1489.

${ }^{21}$ M. Dzugutov, Formation of a dodecagonal quasicrystalline phase in a simple monatomic liquid, Phys. Rev. Lett. 70 (1993) 2924.

${ }^{22}$ A. Quandt and M. P. Teter, Formation of quasiperiodic patterns within a simple twodimensional model system, Phys. Rev. B 59 (1999) 8586.

${ }^{23}$ A. Skibinsky, S. V. Buldyrev, A. Scala, S. Havlin, and H. E. Stanley, Quasicrystals in a monodisperse system, Phys. Rev. E 60 (1999) 2664.

${ }^{24} \mathrm{M}$. Engel and H-R. Trebin, Self-assembly of monatomic complex crystals and quasicrystals with a double-well interaction potential, Phys. Rev. Lett. 98 (2007) 225505.

${ }^{25}$ T. Dotera and T. Gemma, Dodecagonal quasicrystal in a polymeric alloy, Philos. Mag. 86 (2006) 1085. 
${ }^{26}$ R. Lifshitz and D. M. Petrich, Theoretical model for faraday waves with multiple-frequency forcing, Phys. Rev. Lett. 79 (1997) 1261.

${ }^{27}$ H. Davenport and K. Mahler, Simultaneous diophantine approximation, Duke Math. J. 13 (1946) 105.

${ }^{28}$ R. Lifshitz and H. Diamant, Soft quasicrystals-why are they stable? Philos. Mag. 87 (2007) 3021.

${ }^{29}$ K. Jiang, P. Zhang, and A. C. Shi, Theory of emergence and stability of quasicrystals, In preparation.

${ }^{30} \mathrm{P}$. Zhang and X. Zhang, An efficient numerical method of Landau-Brazovskii model, J. Comput. Phys. 227 (2008) 5859.

${ }^{31}$ K. Jiang, C. Wang, Y. Huang, and P. Zhang, Discovery of new metastable patterns in diblock copolymers, Commun. Comput. Phys. 14 (2013) 443.

${ }^{32}$ M. Frigo, and S. Johnson, FFTW: An adaptive software architecture for the FFT, ICASSP Conf. Proc. 3 (1988) 1381.

${ }^{33}$ P. M. Chaikin and T. C. Lubensky, Principles of Condensed Matter Physics, Cambridge University Press, 1995. 